\title{
Images - Isolated caval recurrence of renal cell carcinoma
}

Justin D. Oake, MD ${ }^{1}$; Julie A. Semenchuk ${ }^{2}$; Darrel E. Drachenberg, MD, FRCSC ${ }^{1}$

${ }^{1}$ Section of Urology; ${ }^{2}$ Max Rady College of Medicine; University of Manitoba, Winnipeg, MB, Canada

Cite as: Can Urol Assoc J 2018 July 24; Epub ahead of print. http://dx.doi.org/10.5489/cuaj.5245

Published online July 24, 2018

$* * *$

\section{Introduction}

Renal cell carcinoma (RCC) is unique in that it has a biological propensity for vascular invasion. Approximately $10 \%$ of RCC cases present with venous tumor thrombus involving the renal vein or inferior vena cava (IVC) ${ }^{1,2}$ and $1 \%$ with a thrombus extending into the right atrium. ${ }^{3}$ Isolated caval recurrence of RCC following radical nephrectomy, however, is a rare event. Although systemic treatment may have a role in managing patients with distant metastases, in the case of local recurrence surgical intervention remains the only effective treatment. ${ }^{3}$

Resection and reconstruction of the IVC for primary or recurrent malignancy is performed infrequently due to the prevalence of concurrent metastasis and mortality risks. Despite this, aggressive management may be associated with longer disease-free survival. ${ }^{4}$ Recurrence in the vena cava is important because surgical intervention is technically challenging, requiring cardiopulomary bypass (CPB) and deep hypothermic circulatory arrest (DHCA) if the thrombus extends above the hepatic veins and into the right atrium. ${ }^{5}$ Beyond this, the presence of a venous thrombus is associated with risks such as emboli and venous congestion. ${ }^{3}$ There is little information regarding operative management of recurrent tumor thrombi, due to the rareness of its occurrence. We present a case of RCC with isolated caval recurrence 15 years following a radical nephrectomy. Surgical resection of the thrombus with CPB and DHCA was successfully performed.

\section{Case report}

A 52-year-old male presented initially with gross hematuria and right lower quadrant pain radiating to his testicles. A computed tomography (CT) scan of the abdomen and pelvis described a $12 \mathrm{~cm}$ right renal mass with tumor invasion into the renal vein. There was no evidence of metastatic disease. The patient subsequently underwent a right radical nephrectomy with a renal vein tumor thrombectomy. Tumor invaded the right renal vein and venous wall. Tumor was also seen grossly invading into Gerota's fascia. The IVC appeared patent. Pathology described a 14 x 11 x 11cm right renal mass which was well to moderately differentiated RCC, 
clear cell, with some papillary architecture. Following 5 years of follow-up without recurrence of disease, the patient was discharged back to their family physician.

Ten years later the patient presented to the emergency department complaining of a few weeks of right lower quadrant pain. He was otherwise well. An abdominal ultrasound identified a large thrombus in the upper IVC, approximately $11.5 \mathrm{~cm}$ in length (arising around the location of the right renal vein). The thrombus appeared to change in position with the cardiac cycle. Doppler studies demonstrated flow within and around the thrombus. A CT scan confirmed presence of the IVC mass extending to the intrahepatic IVC. Extension into the right atrium could not be determined. It also demonstrated a $4.5 \times 2.0 \mathrm{~cm}$ mass posterior to the IVC in the renal fossa. A magnetic resonance imaging (MRI) angiogram (Fig. 1) and 3D reconstruction demonstrated thrombus to the level of the right atrium (Fig. 2).

Taking into account the new caval recurrence, the patient was taken for a laparotomy, median sternotomy, and CPB with DHCA. The IVC thrombus, renal vein stump, and paracaval mass were removed en bloc (Fig. 3). A 20mm polytetrafluoroethylene (PTFE) graft was placed in the caval defect because of the circumferential caval wall invasion.

Frozen section taken during the operation showed RCC, clear cell variant. Final pathology of the posterior caval mass and caval thrombus demonstrated clear cell, Fuhrman grade II/IV, skeletal muscle fibers were invaded indicating likely invasion of the psoas and/or paraspinal muscles posterior to the IVC. Paracaval lymph nodes were negative for RCC. The procedure was well tolerated and he recovered appropriately. Subsequent follow-up CT scans of the abdomen and pelvis showed no evidence of recurrence at follow-up of 60 months. Metastatic work-up was also negative at 60 months. Ultrasound also demonstrated patency of his graft, without thrombus.

\section{Discussion}

There are very few documented cases in the literature surrounding isolated caval recurrence of RCC following nephrectomy. The results of our literature review demonstrated that surgical management of the recurrent tumor thrombus is complex, and often multidisciplinary. Other services involved included cardiothoracic surgery, general surgery, hepatobiliary surgery, and vascular surgery. Compared with prior reports, our case demonstrated different findings with respect to the timing of recurrence, where the majority of previous experiences have shown a range of 6-48 months from nephrectomy to the development of recurrent tumor thrombus. In RCC patients with isolated caval recurrence, several surgical methods have been discussed. Regardless of the surgical intervention, most reports emphasized technical difficulty. Given the involvement of tumor thrombus invading up to the level of the right atrium, we felt a combined approach including open midline incision, median sternotomy, and CPB with DHCA would most likely allow successful removal of the tumor and importantly minimize intra-operative complications. 


\section{Conclusion}

While RCC is the sixth and eleventh most common cancer diagnosed in men and women, respectively, ${ }^{6}$ concomitant RCC with isolated caval recurrence following nephrectomy is incredibly rare. Only 26 cases have been reported. Surgery is challenging and usually requires caval replacement and CPB with DHCA if thrombus is extending into the right atrium. Although our experience may justify close long-term follow-up surveillance for RCC patients with T3 disease and venous wall invasion, this may be more a consequence of the biology of this specific cancer and it is therefore difficult to make any inferences about management for cases going forward. 


\section{References}

1. Montie JE, el Ammar R, Pontes JE et al. Renal cell carcinoma with inferior vena cava tumor thrombi. Surg Gynecol Obstet 1991;173:107-15.

2. Marshall VF, Middleton RG, Holswade GR, Goldsmith EI. Surgery for renal cell carcinoma in the vena cava. J Urol 1970;103:414-20.

3. Boorjan SA, Sengupta S, Blute, ML. Renal cell carcinoma: vena caval involvement. BJU Int. 2007;99(5 Pt B):1239-1244.

4. Smaldone MC, Cannon Jr GM, Hrebinko RL. Resection of recurrent inferior vena cava tumor after radical nephrectomy for renal cell carcinoma. Urology 2006;67,1084.e5-7.

5. Hatcher PA, Anderson EE, Paulson DF, Carson CC, Robertson JE: Surgical management and prognosis of renal cell carcinoma invading the vena cava. J Urol 1991;145(1):20-3

6. Rendon RA, Kapoor A, Breau R. Surgical management of renal cell carcinoma: Canadian Kidney Cancer Forum Consensus. Can Urol Assoc. 2014;8(5-6):e398-e412.

7. Smith RB. Long-Term Survival of a Vena Caval Recurrence of Renal Cell Carcinoma. $J$ Urol. 1981;125(5):575-578.

8. Finkelstein MP, Drinis S, Tortorelis DG, et al. Recurrence of Renal Cell Carcinoma with Extensive Vena Caval Thrombus Three Years after Radical Nephrectomy. Urol Int. 2002;68: 199-201.

9. Minervini A, Salinitri G, Lera J, et al. Case Report Solitary floating vena caval thrombus as a late recurrnece of renal cell carcinoma Case report. Int J Urol. 2004;11:239-242.

10. Horger DC, Bissada NK, Curry NS, et al. Isolated late recurrence of renal cell carcinoma in the inferior vena cava. Can J Urol. 2004;11(5):2467-2469.

11. D’Arggio L, Pennisi M, Pepe P. Isolated Local Recurrence of Renal Neoplasm with Caval Involvement 16 Years After Radical Nephrectomy. Arch Esp Urol. 2005;10:1.0931.094.

12. Li G, Zhang Z, Xie D, et al. Surgical resection of recurrent inferior vena cava tumor following radical nephrectomy for renal cell carcinoma: A case report. Oncol Lett. 2015;10:111-114.

13. Parker WP, Boorjian SA, Zaid HB, et al. Surgical Management and Oncologic Outcomes of Recurrent Venous Tumor Thrombus after Prior Nephrectomy for Renal Cell Carcinoma. Eur Urol Focus. 2016;2(6):625-630. 
Fig. 1. A magnetic resonance imaging angiography demonstrating tumour thrombus to the level of the right atrium.

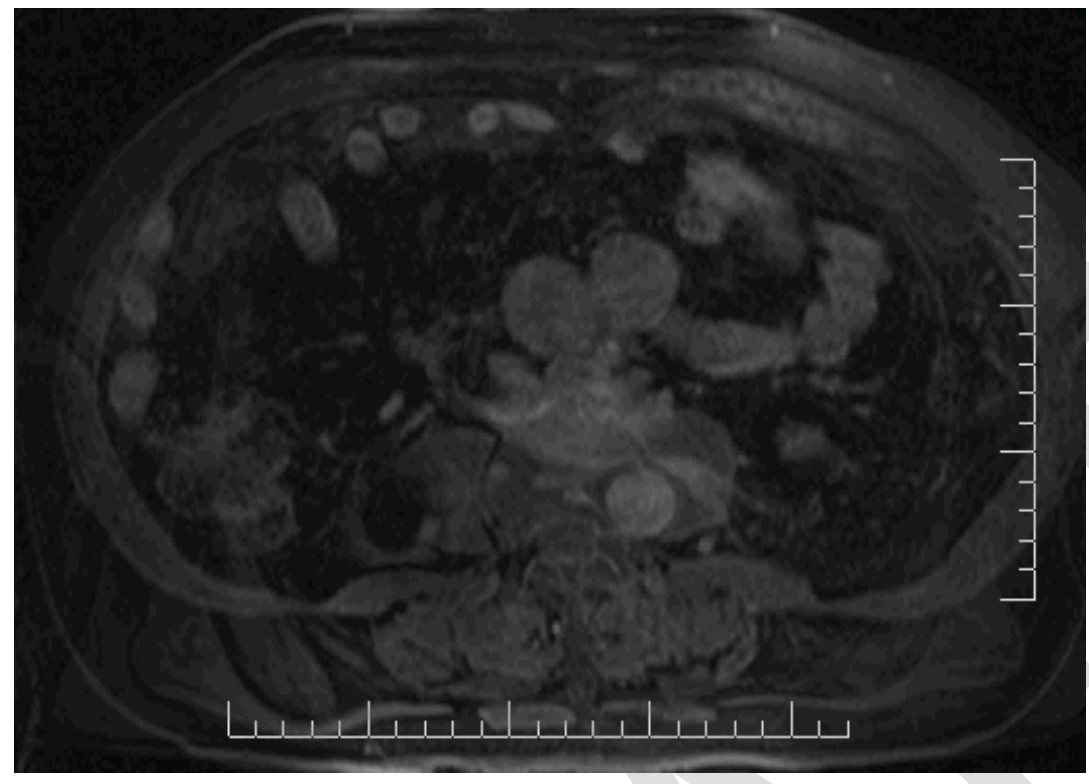

Fig. 2. A magnetic resonance imaging with 3D reconstruction demonstrating tumor thrombus extending to the intrahepatic inferior vena cava.

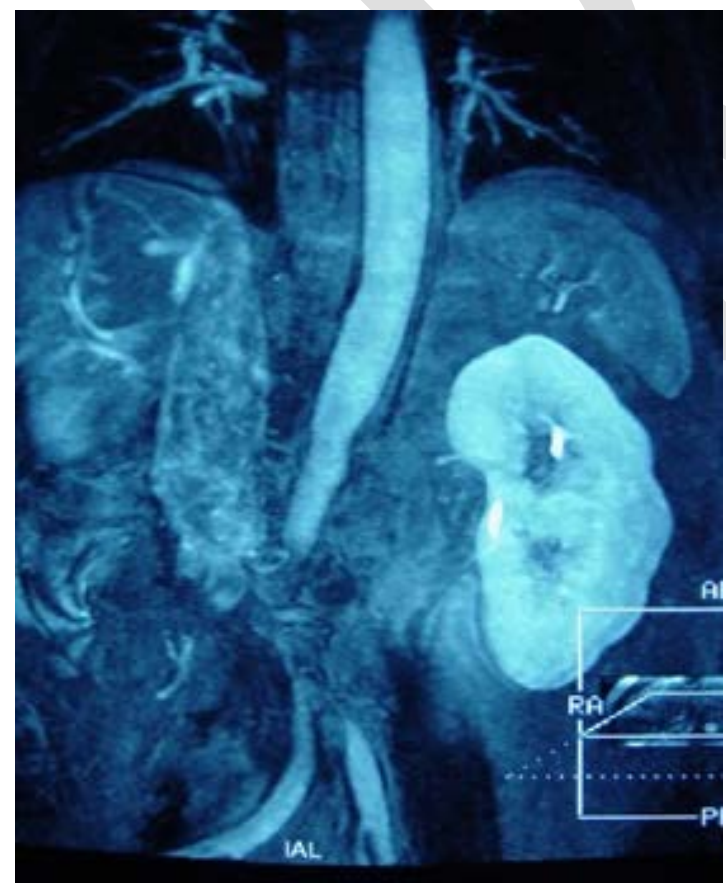


Fig. 3. Inferior vena cava tumour thrombus, renal vein stump, and paracaval mass. Probe transverses the vena cava.

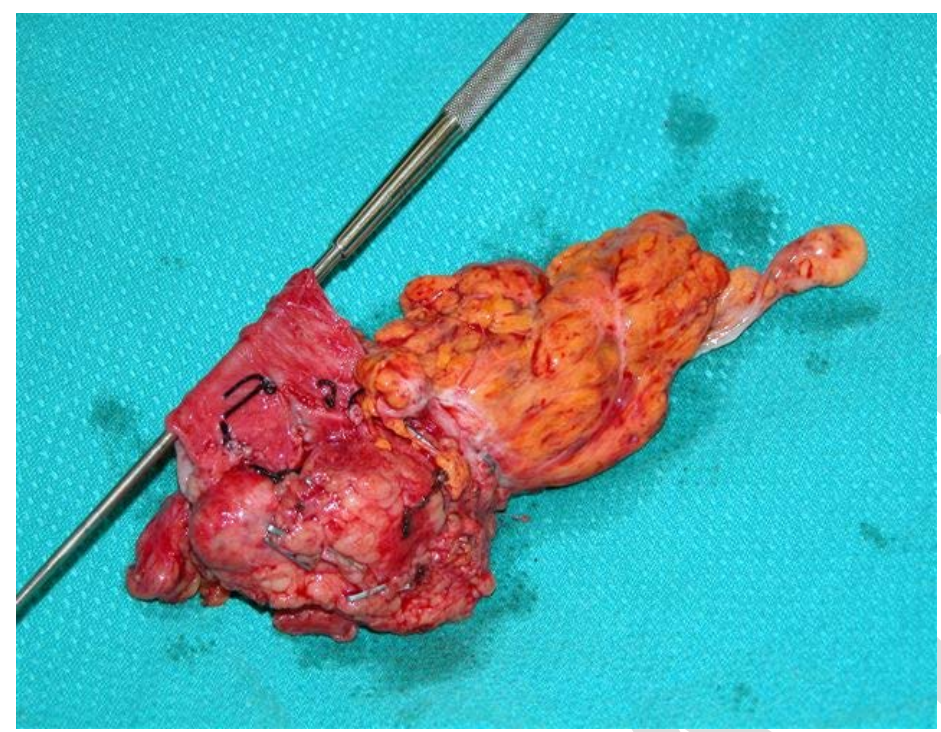

